\title{
The study of the structures of the biomacromolecules in solution with correlated X-ray scattering
}

\author{
Shengjun Liu $^{1,2, a}$ \\ ${ }^{1}$ Department of electronic and information engineering, Xiamen City University, Xiamen 361008, \\ China; \\ ${ }^{2}$ Department of Applied Physics, Stanford University, Stanford CA 94305, United States. \\ ashengjun@xmcu.cn
}

Keywords: The correlated X-ray scattering (CXS), filtering, clusters, correlation.

\begin{abstract}
The correlated X-ray scattering (CXS) was introduced and compared with other methods which were used to study the structures of the biomacromolecules in solution, such as nuclear magnetic resonance (NMR), electron microscope (EM) and small-angle x-ray scattering (SAXS). Then a key step which was used to filter the scattering data was put forwarded. And some important clusters were obtained with density based scanning method. Finally, the clusters will lay a good foundation in computing the correlation in future.
\end{abstract}

\section{Introduction}

There are many kinds of methods to study the structures of the biomacromolecules in solution, such as nuclear magnetic resonance (NMR), electron microscope (EM), small-angle x-ray scattering (SAXS) and so on. The NMR can be used to measure the nuclear magnetic resonance spectrum with two dimensions and three dimensions [1]. The three dimensional chemical parameters such as bond distance and bond angle of the different atoms can be computed with the position of the peak of the nuclear magnetic resonance spectrum[2, 3]. Therefore, the structure of the biomacromolecules in solution with three dimensions can be obtained. However, there are some limitations with this method. Firstly, the sensitivity is so low that it needs the high concentration of the solution. Secondly, the resolution ratio is also low and there is the upper bound of molecular weight. Thirdly, the response is so low that limits the stability of the sample. Finally, the weak interaction and the intermediate capture technology are deficient $[4,5]$.

The EM can be applied into record the two-dimension projection of a large number of identical particles. It can also be used to compute the orientation of each particle. In addition, it can be used to reconstruct the three-dimension structure of the particles $[6,7,8]$. But there are also some limitations for it. For example, the molecule weight must be large enough. And the resolution is only about $1 \mathrm{~nm}$. Atomic resolution structure can only be obtained for the high symmetry particles such as icosahedron virus. In addition, the ration of the signal and noise is very low because of the radiation damage of the electrons. Finally, a lot of images have to be recorded with this method [4, 5, 6].

The SAXS method were used more and more recently. There are some distinct advantages. Firstly, the crystals are not needed and the experiment can be done in the solution [9]. Secondly, there are no limitations for the molecular weight and the concentration of the solution. Thirdly, the experiment time is so short that the requirement for the stability of the sample is very low. So it reflects the real state of the biomacromolecules. Fourthly, the equipments are very simple and the in-situ measurement techniques can be used $[4,5]$. However, there are some disadvantages with the SAXS. Because the information which can be obtained with the SAXS is very limited. And it is very difficult to obtain the three-dimension structure information. Only the rough and low resolution information such as the shape, the size, some critical fragment and the spacial relationship of the constituents of the biomacromolecules can be obtained. Because there are not enough standard experiments and structural analysis processes. So the theory of the SAXS is not so perfect compared with the crystallographic diffraction. 


\section{The correlated $X$-ray scattering(CXS)}

The correlated X-ray scattering (CXS) method with which the structure of the biomolecules in solution determined by the X-ray was fist put forward in 1970s by Kam[10]. The model related with the SAXS is not needed. And the time integration patterns from X-ray scattering are not be collected because the particles rotate freely during the scattering. Because of the ultrafast and high-flux free electron laser pulse, the radiation dose which reaches to the molecules of the sample greatly exceeds the limit of the safety radiation dose in crystallographic. And the scattering patterns are recorded before the structure of the sample molecules is damaged [10,11, 12, 13].

So it is perfect to analysis the XFEL scattering data with Kam's theory. Because the scattering occurs in the room temperature and the scattering patterns are recorded before the damage. In addition, the resolution will not be influenced by the damage of the samples. The particles of the sample were frozen by the concentrated X-ray pulses which are shorter than the freely rotating time of the molecules. Thus, the scattering patterns obtained by this method contain the fluctuations of angular intensity. And the fluctuations of angular intensity contain many internal structural information of many molecules which can be obtained by the correlation of the scattering intensity [14]. The information that the correlated scattering data contains is proportional with the cubic of the particles' size [15]. And it is different from the SAXS data with isotropy from which the information obtainded is linear with the particles' size. So in some extent, these information can be used to reconstruct the structure of the biomolecules as Kam putforward. The sum of the angle auto-correlation function recovers the disorder of the directions of many molecules [10, 14]. After that, Kam studied the details of the electron microscopic analyzed from the space correlation. Because the high order correlation measurement was introduced, the rotational uncertainty of the spherical harmonic expansion coefficient in the past research was solved. Hence, the three-dimension reconstruction without heavy atom

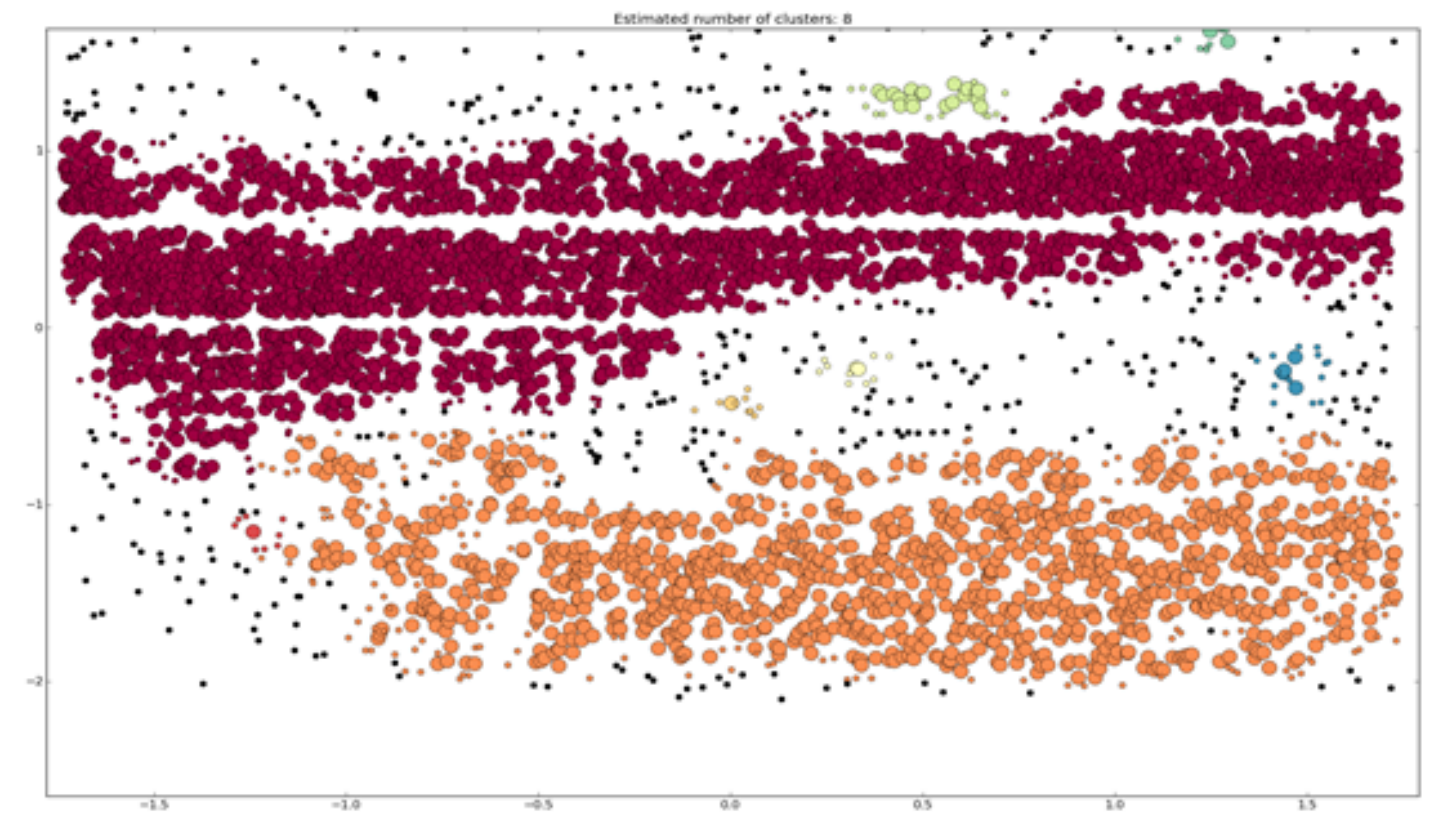

Fig. 1 Eight Clusters

marker can be done. These methods were firstly used in the projection of the two-dimension transmission electron microscope (2DTEM) and the scanning transmission electron microscope (STEM). And then they were used in the three-dimension reconstruction of the virus particles with icosahedron in any direction[14, 16]. 


\section{The data filtering method with density based scanning}

Because there are millions of X-ray scattering patterns for correlated X-ray scattering method. We have to filter the useless scattering patterns and compute the correlation with the good scattering patterns. So it is very important to find a good data filtering method to analyse the data. Firstly, we calculated the sum of scattering intensity, the median of scattering intensity and the variance of scattering intensity. Then, based the density scanning we obtained some clusters of the scattering intensity clusters. Throng filtering some small distinct clusters, we can obtain one or two or three main clusters. For example, we obtained two clusters with red and orange colours in Fig. 1, we obtained red and green colours in Fig. 2 and we obtained one red cluster in Fig. 3 and Fig. 4. So we can filter other scattering intensity.

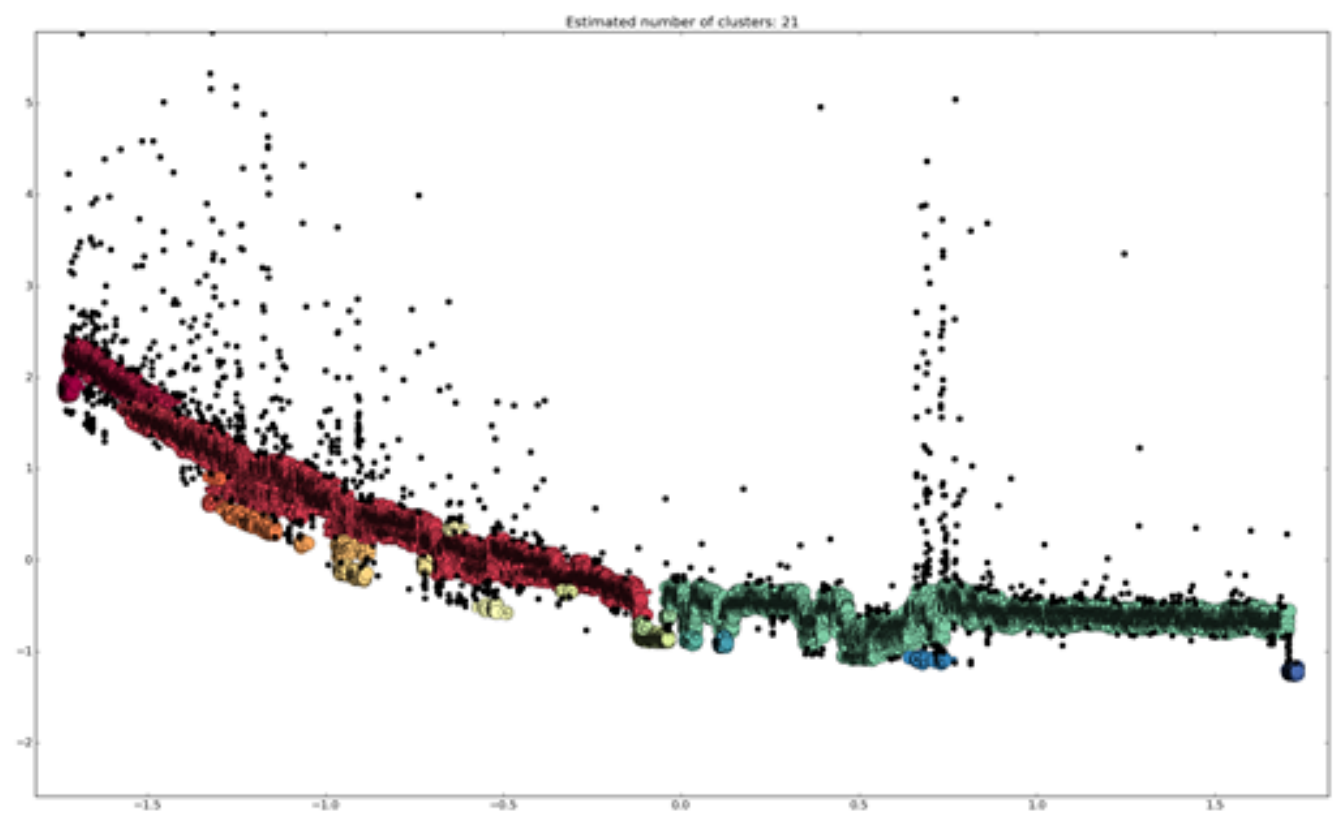

Fig. 2 Twenty-One Clusters

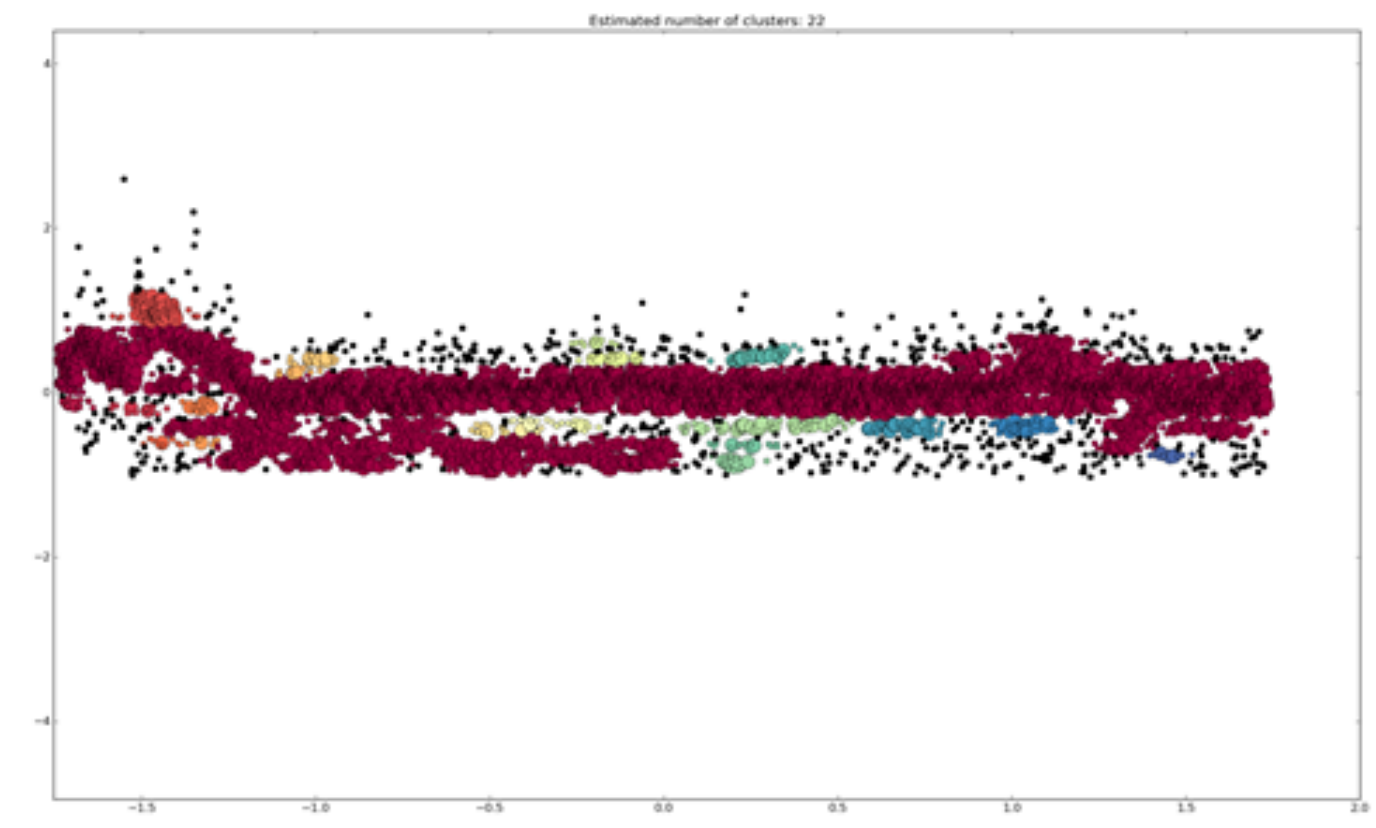

Fig. 3 Seventeen Clusters 


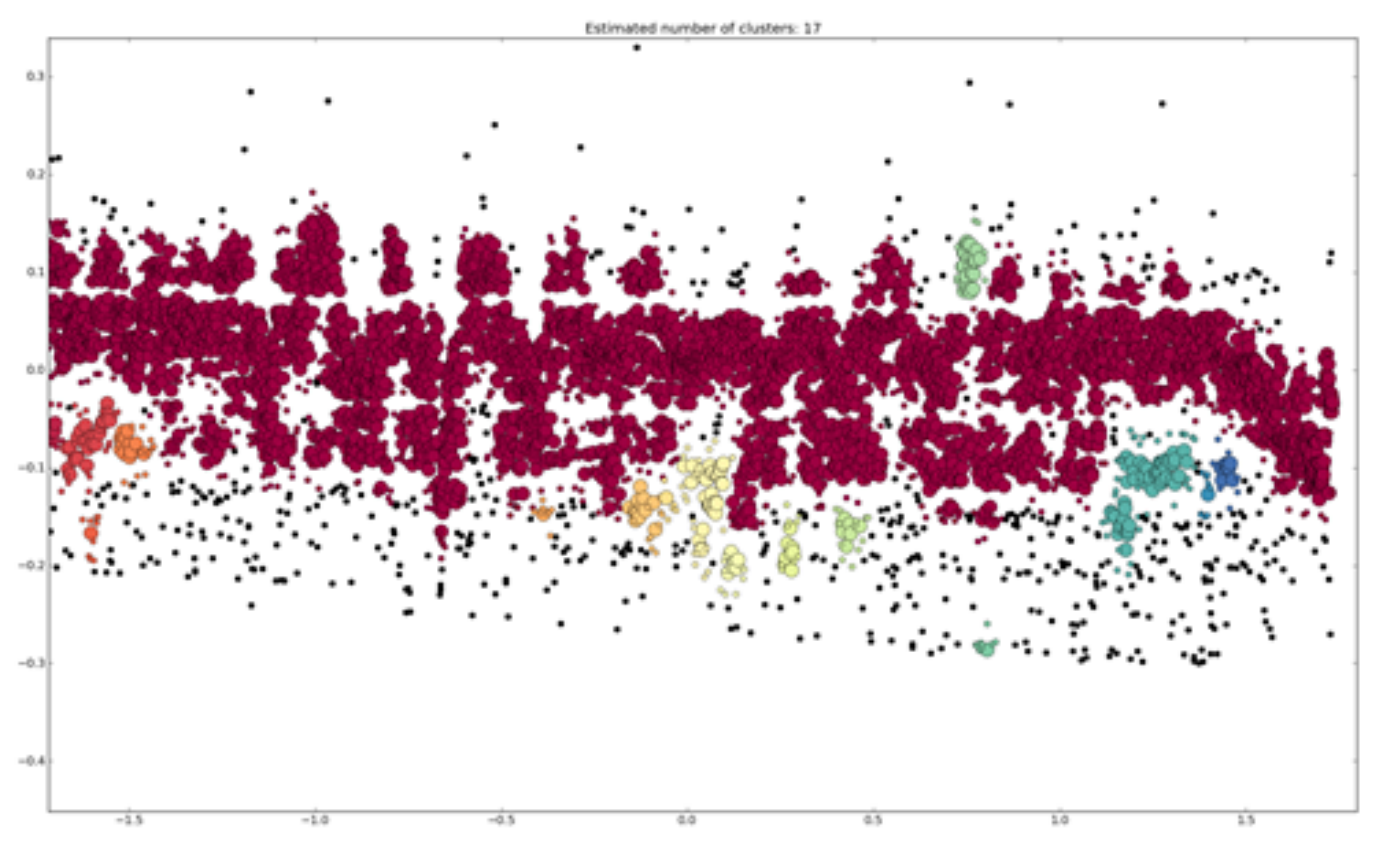

Fig. 4 Twenty-Two Clusters

\section{Summary}

Some methods to study the structures of the biomacromolecules in solution such as nuclear magnetic resonance (NMR), electron microscope(EM), small-angle x-ray scattering (SAXS) were described firstly. Then the correlated X-ray scattering was introduced and the advantages of it were described too. Finally, a good filtering method was putforwarded and some clusters were showed. The density based scanning method will be very usefull for computing the correlation in future. Through the computing the correlation of the scattering intensity, we can obtain the structure information of the biomacromolecules in solution.

Acknowledgements: This research was supported in part by National Nature Science Foundation of China for theoretical physics grant no. 11547238, Technical program of Xiamen City University grant no. KYKJ2014-1, National Insti- tutes of Health research grant 251 R01-GM097463, Stanford NIH Biotech- nology Training grant no. 5T32GM008412-20 and US Department of Ener- gy Office of Science under contract no. DE-AC02-05CH11231.

\section{References}

[1] G. Cornilescu, A L. Didychuk, M L. Rodger, L A. Michael, J E. Burke, E J. Montemayor, A A. Hoskins and S E. Butcher, J Mol Biol 428, 777-789 (2016).

[2] H B. Tan, X Q. Song, H. Yan and H X. Xin, Journal of Molecular Structure 1129, 23-31(2017).

[3] A. L, W L. D, R Y. W, J H. He, X H. Wang, Y X. Sun, Y. Gu and N L. J, Journal of Natural Gas Science and Engineering 37, 29-38 (2017).

[4] E P. Favvas, K L. Stefanopoulos, A. Ch. Mitropoulos and N. K. Kanellopoulos, Microporous and Mesoporous Materials 209, 122-125 (2015).

[5] L. Yao, J. H. Yin, J. L. Li, M. H. Chen, B. Z. Han, G. Mo, H. Zhao, Polymer Testing 53, 7-14 (2016).

[6] G. C. Sneddon, P. W. Trimby, J. M. Cairney, Materials Science and Engineering R 110, 1-12 (2016). 
[7] K. Sakai, H. Takeshita, T. Haraguchi, H. Suzuki, F. Shashi, T. Kume, A. Fukuyama, S. Nonomura, T. Imari, Thin Solid Films 621, 32-35 (2017).

[8] S. K. Sardar, S. K. De, Journal of Microscopy and Ultrastructure 4, 211-221 (2016).

[9] Q. Kuang, J. C. Xu, Y. R. Liang, F. W. Xie, F. Tian, S. M. Zhou and X. X. Liu, Food Hydrocolloids 62, 43-48 (2017).

[10] Z. Kam, Macromolecules 10, 927-934 (1977).

[11] R. Neutze, R. Wouts, D. van der Spoel, E. Weckert, and J. Hajdu, Nature 406, 752-757 (2000).

[12] M. Bergh, G. Huldt, N. T1 ^mneanu, F. R. N. C. Maia and J. Hajdu, Quart. Rev. Biophys. 41, 181-204 (2008).

[13] A K. Richard, J. phys. B: At. Mol. Opt. Phys. 45, 223001 (2012).

[14] D. Starodub et al. Nat. Commun. 3, 1276 (2012).

[15] M. Derek, J. L. Thomas, J. M. Sung, S. Jonas, Clément Levard, Herschel Watkins, Aina E. Cohen, Michael Soltis, Shirley Sutton, James Spudich, Vijay Pande, Daniel Ratner, Sebastian Doniach, Phil. Trans. R. Soc. B 369, 20130315 (2014).

[16] Z. Kam, I. Gafni and M. Kessel, Ultramicroscopy 7, 311-320 (1982). 